\title{
¿Cómo es un mejor amigo o mejor amiga? Características de los vínculos de amistad en niños y niñas de Argentina
}

\section{How is a best friend? Friendship's characteristics in Argentine children}

\author{
Carolina Greco ${ }^{1}$ \\ ${ }^{1}$ Consejo Nacional de Investigaciones Científicas y Técnicas (CONICET), \\ Instituto de Ciencias Humanas Sociales y Ambientales (INCIHUSA), \\ Universidad del Aconcagua, Argentina
}

\begin{abstract}
Resumen. Los objetivos fueron indagar el concepto de mejor amigo en escolares y explorar la comprensión del mejor amigo como factor protector en situaciones de maltrato entre pares. Se optó una metodología cualitativa y la indagación mediante grupos focales. Participaron 85 escolares de $4^{\circ}$ a $7^{\circ}$ grado $(M=10.68, D T=1.34)$. Se realizó el análisis del método comparativo constante. Las categorías emergentes fueron: compañerismo, conflictos, fraternidad, fidelidad, confianza, ayuda, emociones positivas y aspectos morales, manifestaciones de violencia y búsqueda de diálogo con el agresor, contención afectiva hacia la víctima y recurrencia al docente. Diferentes conceptualizaciones se interpretan considerando el desarrollo cognitivo y socioemocional. La amistad es un recurso psicosocial para amortiguar efectos del rechazo y propiciar el desarrollo socioemocional.
\end{abstract}

Palabras clave. Amistad, infancia, mejor amigo, desarrollo socioemocional.

Abstract. The aims were to inquire the concept of best friend in school children and to explore the best friend concept as a protective factor against situations of mistreatment between peers. A qualitative methodology was chosen and focus groups made. The sample was made up of 85 schoolchildren, from 4 th to 7 th grade $(M$ $=10.68, S D=1.34)$. The analysis was carried out via constant comparative method. The emergent categories were: companionship, conflicts, fraternity, fidelity, trust, help, positive emotions and moral aspects, manifestations of violence and search for dialogue with the aggressor, affective containment towards the victim and recourse for teacher support. Different conceptualizations are interpreted considering cognitive and socioemotional development. Friendship is a psychosocial resource to cushion the effects of rejection and foster socioemotional development.

Keywords. Friendship, childhood, best friend, socioemotional development.

${ }^{1}$ Carolina Greco. Consejo Nacional de Investigaciones Científicas y Técnicas (CONICET), Instituto de Ciencias Humanas Sociales y Ambientales (INCIHUSA), Universidad del Aconcagua, Argentina. Direccion Postal: Av. Ruiz Leal S/N . Parque General San Martin. Ciudad Mendoza. CP 5500. E-mail: cgreco@mendoza-conicet.gob.ar

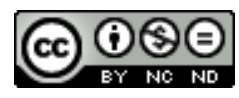

Esta obra está bajo una licencia de Creative Commons Reconocimiento-NoComercial-SinObraDerivada 4.0 Internacional. 


\section{Introducción}

Los vínculos de amistad que los niños y las niñas establecen con sus pares constituyen un importante contexto para el desarrollo socioemocional en la infancia. En el marco de este contexto, los niños y las niñas adquieren habilidades, comportamientos, actitudes, valores y experiencias que inciden y promueven su funcionamiento afectivo, social y cognitivo a lo largo de la vida (Rubin, Bukowski, \& Parker, 2007; Vitaro, Boivin, \& Bukowski, 2009).

Fuertes lazos afectivos positivos que inciden en el desarrollo socioemocional definen a la amistad (Hartup \& Stevens, 1997; Hinde, 1987). Esta se caracteriza por el compañerismo, una historia compartida y el afecto mutuo (Rubín \& Bowker, en prensa). Es probable que la conceptualización más antigua y completa de amistad se encuentre en la escritura de Aristóteles. Él afirmaba que el verdadero objetivo de la amistad era la bondad. Desde su punto de vista, bondad y amistad estaban entrelazadas. De esta manera, la amistad era el componente central del modelo de ética de Aristóteles y la ética era un rasgo central de la amistad (Saldarriaga, Bukowski, \& Greco, 2015).

Según Kancyper (2014), la amistad es una relación de hermandad elegida que se caracteriza por una admiración recíproca en la cual se conjugan varias cualidades como confianza, lealtad, honestidad, compasión, solidaridad, compromiso, empatía recíproca, ternura y alegría. Al respecto, refiere que los componentes positivos en la amistad prevalecen sobre los negativos. Así, por ejemplo, la admiración está sobre la envidia; la empatía sobre la indiferencia; el resentimiento, los celos y la competencia lúdica sobre la rivalidad. La falta de equilibrio entre estos elementos pone en juego la trama vincular y la calidad de la amistad, generando situaciones conflictivas pero también la resolución y reflexión de tales conflictos.

En cuanto a las teorías que abordan los vínculos de amistad, es importante destacar que coexisten varias; ya sea desde la sociología, psicología o antropología, que permiten describir tal fenómeno (Villalobos Carrasco, 2015). Hartup (2009) refiere que son diversos los marcos teóricos a los cuales remitirse cuando se trata de estudiar las relaciones entre pares o entre iguales.

Entre los marcos teóricos, el autor alude a la teoría psicodinámica, cuyo máximo exponente es Freud (1997). También, incluye dentro de las teorías psicodinámicas a Sullivan (1953) y a Peter Blos (1979); el primero intenta explicar, desde el modelo de relaciones interpersonales, la personalidad. Otra de las teorías a través de las cuales se comprenden las relaciones entre pares es la teoría del apego (Bowlby, 1958; Ainsworth, 1989). Entre las teorías cognitivas, Hartup menciona a Selman (1980), quien estudió el desarrollo del pensamiento acerca de la amistad y propuso que tal desarrollo está vinculado al proceso de toma de perspectiva en la infancia. Del mismo modo, explica la teoría de los sistemas del comportamiento que conjuga la teoría interpersonal de Sullivan (1953), las teorías cognitivas y la teoría del apego (Collins, Welsh, \& Furman, 2009) y, finalmente, menciona la teoría del intercambio social (véase Thibaut \& Kelly, 1959). Al respecto, Villalobos Carrasco (2015) refiere que se han utilizado los aportes de la Psicología del Desarrollo, de la Psicología de la Personalidad y Psicología Social para poder comprender e investigar los estudios de la amistad. 
En este trabajo se consideraran las contribuciones de grandes teóricos dedicados al estudio de la amistad: Sullivan (1953), Selman (1980), Hartup (2009) y Bukowski y Sippola, (2005); estos últimos investigadores contemporáneos del estudio de la amistad.

Siguiendo, entonces, a Selman (1980), quien estudió el concepto sobre la amistad a lo largo de cinco etapas superpuestas, halló que la mayoría de los niños/as en edad escolar se encuentra en la etapa "dos", caracterizada por una amistad recíproca basada en intereses propios (Papalia, Wendkos, \& Duskin, 2010). La amistad en esta etapa se determina por la cooperación y la ayuda recíproca puestas al servicio del logro de un objetivo (Fuentes Rebollo \& Merelo Zabal, 1992). Sin embargo, algunos niños y niñas de edad escolar, pero de edades mayores (9 años y más), atraviesan la etapa "tres". En esta etapa los niños y las niñas son parte de una reciprocidad mutua; en este sentido, se incorpora algo más que hacer algo por la otra persona, predominando relaciones íntimas y mutuamente compartidas que exigen exclusividad, aspecto que en algunas ocasiones conlleva a sentimientos de celos y posesión (Hartup, 2009; Papalia, Wendkos, \& Duskin, 2010).

A partir de los 12 años, y ya finalizando la escolaridad primaria, los niños y las niñas entrarían en la etapa denominada por Selman (1980) de "Independencia Autónoma", en la cual se concibe la relación de amistad en ambos sentidos de dependencia e independencia; son capaces de apoyar, confiar y dar, pero también de soltar (Papalia, Wendkos, \& Duskin, 2010). La intimidad, cercanía y lealtad cobran valor en la preadolescencia y adolescencia, aspectos que van de la mano del desarrollo de un pensamiento lógico formal y abstracto (Papalia, Wendkos, \& Duskin, 2010).

La descentración y disminución del egocentrismo de los niños y las niñas en edad escolar permiten comprender las relaciones interpersonales con base en la reciprocidad. Así, la posibilidad de realizar evaluaciones recíprocas conlleva a pensar y consolidar la amistad con base en la confianza recíproca. Al respecto, Hartup (2009) refiere que la reciprocidad constituye una característica importante en los vínculos de amistad en todas las etapas del desarrollo, lo que cambia con la edad serían las formas en que dicha reciprocidad se manifiesta en los diferentes comportamientos sociales.

La experiencia de reciprocidad en los vínculos de amistad proporciona a los niños y las niñas una sensación de bienestar y de validación del yo, que a su vez tiene un efecto relevante en el desarrollo del concepto de sí mismo (Sullivan, 1953). Este es un punto crucial que Sullivan (1953) enfatizó en la temática de la amistad en la infancia, vale decir, la importancia y contribución que tiene para el desarrollo del sí mismo el vínculo de amistad con un mejor amigo. Este vínculo de cercanía con el mejor amigo ofrece la oportunidad de reflejar la apreciación y valoración que los amigos hacen de uno mismo y cuán valioso es uno para los amigos, aspecto que contribuye a la valoración del self. Es importante aclarar que este proceso de validación, de respeto mutuo y reciprocidad que plantea Sullivan (1953), no se daría en una relación de amistad casual, sino que se da en la relación de cercanía que se establece con el mejor amigo.

Las experiencias positivas con el mejor amigo podrían ser tan poderosas y fuertes que permitirían a los niños y adolescentes poder superar situaciones disfuncionales y conflictivas, ya sea en el entorno familiar o en el contexto las relaciones entre los pares. 
Una de las funciones que la literatura destaca en la teoría de Sullivan (1953) es la función de los vínculos de amistad como factores protectores frente a situaciones de riesgo o de vulnerabilidad, dentro de los que resalta esta característica de seguridad y protección que emana de los vínculos de amistad con el mejor amigo.

En el contexto de las relaciones entre pares, hay evidencia empírica de que los vínculos de amistad son un factor protector frente a situaciones de intimidación y maltrato entre pares (Bukowski, Motzoi, \& Meyer, 2009). Al respecto, Hodges, Boiven, Vitaro y Bukowski (1999) realizaron un estudio con niños/as en el cual evaluaron la función protectora del mejor amigo en situaciones de victimización entre pares. Ejemplos de problemas de índole interna propios de quienes han sufrido el ciclo de la victimización con sus pares que se hallaron son: el retraimiento social, tristeza, sentimientos de soledad, ansiedad fueron atenuados al contar con un mejor amigo. Los resultados también señalaron que comportamientos agresivos fueron disminuidos hacia aquellos escolares que tenían un mejor amigo.

En línea con lo planteado, Saldarriaga, Bukowski y Greco (2015), explican que niños y niñas con comportamientos agresivos podrían sentir temor por parte de los amigos de la víctima y, en ese sentido, disminuir el comportamiento agresivo. Además, los niños y las niñas que tienen amigos suelen estar en compañía de otros y, por lo tanto, no son elegidos como objetivos de victimización. Los autores sugirieron que los niños y las niñas que tienen amigos podrían recibir consejos de ellos sobre cómo enfrentar la intimidación por parte de los pares. Del mismo modo, Kochenderf y Ladd (1996) refirieron que el vínculo de amistad con el mejor amigo puede atenuar la victimización a través de estrategias de comunicación hacia el docente por parte del mejor amigo.

Estudios también refieren que las amistades no solo tienen el potencial de proteger a los individuos en situaciones de vulnerabilidad, sino que también promueven un desarrollo positivo en la infancia. Así, por ejemplo, estudios que han explorado la relación entre la amistad y la felicidad a lo largo del desarrollo refieren que estas dos variables están relacionadas de manera bidireccional (Demir \& Özdemir, 2010; Demir, Orthel, \& Andelin, 2013; Holder \& Coleman, 2008; Saldarriaga, Bukowski, \& Greco, 2015).

Un estudio sobre qué hace felices a los niños y niñas argentinas halló que las relaciones de amistad en la infancia son una de las fuentes más importantes de felicidad y de emociones positivas tales como la alegría, la diversión y el amor recíproco (Greco \& Ison, 2014). Las relaciones de amistad implican una fuerte experiencia emocional positiva como la aceptación, la intimidad, la lealtad, la seguridad y altos niveles de autoestima, por eso contribuyen al bienestar (Bukowski, Hoza, \& Boivin, 1994; Bukowski \& Sippola, 2005; Rubin, Coplan, Chen, Bowker, \& McDonald, 2011).

Con base en los planteamientos teóricos señalados surgen, entonces, las siguientes interrogantes: ¿qué caracteriza a un mejor amigo según los niños y las niñas argentinas que se encuentran en la escolaridad primaria?, ¿es el mejor amigo un factor protector en situaciones de victimización entre pares para los niños argentinos en la escolaridad primaria? Si bien hay estudios e investigaciones que abordan dichos cuestionamientos a nivel internacional, no se han encontrado estudios de tales características en muestras 
argentinas y desde un enfoque cualitativo. Al respecto, Hendrick \& Hendrick (2000) refieren que son pocos los estudios sobre las relaciones interpersonales cercanas o íntimas realizadas desde el enfoque de investigación cualitativo; la mayoría de este tipo de temática se ha enfocado desde la metodología cuantitativa. En línea con lo planteado, Potocnjak, Berger y Tomicic (2011) explican que, en la temática de las relaciones entre pares, los estudios se han focalizado en describir el fenómeno, su prevalencia y variables asociadas desde un enfoque cuantitativo. La importancia de este estudio radica, entonces, en su acercamiento cualitativo.

El estudio de la amistad desde la perspectiva cualitativa permitirá conocer su naturaleza con base en la subjetividad y referencia contextual. Tal perspectiva permitirá explorar nuevos aspectos de la dinámica vincular en la infancia y generar información que permita desarrollar intervenciones, desde la voz de los propios niños y niñas, adecuadas a los contextos de referencia.

Así, los objetivos de este trabajo fueron: 1) Indagar el concepto de mejor amigo que presentan los niños y las niñas que concurren a una escuela de gestión estatal de $4^{\circ}, 5^{\circ}, 6^{\circ}$ y $7^{\circ}$ grado; y 2) Explorar la comprensión que tienen los escolares de $4^{\circ}, 5^{\circ}, 6^{\circ}$ y $7^{\circ}$ grado sobre el concepto del mejor amigo como factor protector frente a situaciones de maltrato entre pares.

\section{Método}

Tipo de estudio

Se optó por una metodología de investigación cualitativa, utilizando el enfoque de la teoría fundamentada (Glaser \& Strauss, 1967).

\section{Participantes}

Participaron en el estudio 85 escolares de entre 9 y 14 años de edad $(M=10.68, D T$ $=1.34$ ), desde $4^{\circ}$ a $7^{\circ}$ grado. El $48.2 \%$ fueron niñas y el $51.8 \%$ varones. Asistían a una escuela primaria de gestión estatal de Mendoza.

\section{Instrumentos}

Grupos focales; se trata de una técnica que propicia la exploración de un tema a partir de la interacción entre los participantes. Los grupos focales son útiles cuando se quiere conocer no solo lo que la gente piensa sino también cómo y por qué piensa como lo hace (Kornblit, 2004).

Los grupos focales se realizaron por grado, conformado un corpus de 19 grupos focales en total ( 5 en $4^{\circ}$ grado; 4 en $5^{\circ}$ grado; 6 en $6^{\circ}$ grado y 4 en $7^{\circ}$ grado). La cantidad de grupos focales estuvo dada por el criterio de saturación muestral. Cada grupo focal estuvo conformado por un total de entre 3 y 6 escolares.

Los grupos focales se realizaron en horario escolar y su duración fue de 80 minutos, con un descanso de 10 minutos correspondiente al recreo escolar. Los primeros 20 minutos estuvieron dedicados al encuadre de trabajo y la creación del rapport, necesarios para llevar a cabo la actividad. Cada grupo focal estuvo compuesto por un moderador (investigadora 
asistente de CONICET y especialista en psicología clínica) y un observador no participante (estudiante avanzado de la carrera de Psicología), quien había recibido en entrenamiento pertinente para dicha tarea.

Las preguntas guía fueron: 1) ¿Qué sería un mejor amigo para ustedes? ¿Cómo es?, ¿qué hace?; 2) ¿Tienen un mejor amigo acá en la escuela? (Sí, No) ¿Por qué?; 3) Aquellos que tienen un mejor amigo acá en la escuela: ¿sienten que pueden contar con él o con ella cuando hay algunas situaciones de burlas y/o malos tratos entre ustedes? ¿Cómo?, ¿en qué cosas?

\section{Procedimiento}

Se solicitó permiso a las autoridades escolares y el consentimiento informado a los padres y/o tutores por escrito. La presentación con los escolares se realizó por grado a través de una técnica lúdica que permitió establecer el rapport y clima de trabajo apropiado. A los escolares se les explicó que todo el trabajo no sería evaluado con calificaciones y que ellos podían decidir dejar de participar si así lo quisieran. Estos aspectos fueron importantes para evitar la deseabilidad social y promover el libre consentimiento de participar. Se les solicitó el consentimiento para las grabaciones de voz en los grupos focales.

Procedimientos para el análisis de los datos

Predominó una codificación abierta y axial (Kornblit, 2004) que permitió identificar el espectro y variedad de temas emergentes en relación con las características del mejor amigo y al concepto del mejor amigo como factor protector frente a situaciones de maltrato entre pares. Se trabajó con un manual de categorías construido con base en los objetivos del trabajo, las contribuciones teóricas y el análisis de interpretación hermenéutica del contenido de respuestas por parte del investigador y colaboradores. En las tablas 1 y 2 se incluye el esquema de las categorías.

Se utilizó el índice de $x$ de Cohen (Cohen, 1960) para evaluar el acuerdo entre jueces, juzgando como aceptables valores de $x$ mayores o iguales a .70 (Landis $\&$ Koch, 1977). Para tal fin, se utilizó una grilla de respuesta tipo Likert con valores del 1 al 3, donde el valor 1 fue inadecuado, 2 medianamente adecuado y 3 adecuado. Los jueces expertos fueron dos investigadores de CONICET especialistas en desarrollo socioemocional en la infancia. Se registraron buenos índices de acuerdo entre los jueces intervinientes en las siguientes categorías: compañerismo $(\kappa=.83)$, conflicto $(\kappa=.72)$; fraternidad $(\kappa=$ $.92)$; semejanza $(\kappa=.90)$; confianza $(\kappa=.75)$; ayuda $(\kappa=.84)$; aspectos emocionales y morales $(\kappa=.90)$; rol protector: manifestaciones de violencia hacia el agresor $(\kappa=.80)$; rol protector: búsqueda de dialogo con el agresor $(\kappa=.75)$; rol protector: estrategia de ignorar al agresor o agresores $(\kappa=.73)$; rol protector: contención afectiva por medio de la palabra hacia la víctima $(\kappa=.78) \mathrm{y}$, finalmente, para la categoría rol protector: búsqueda de apoyo en el docente $(\kappa=.92)$.

\section{Resultados}

Se observa, en la tabla 1 que emergen del discurso de los niños y las niñas nueve categorías: compañerismo, conflictos, fraternidad, fidelidad, semejanzas, confianza, ayuda, emociones positivas y aspectos morales al caracterizar al mejor amigo. 
Tabla 1

Esquema de categorías empleadas en el análisis del concepto de mejor amigo

\begin{tabular}{|c|c|c|}
\hline Categoría & Definición & Ejemplo \\
\hline Compañerismo & $\begin{array}{l}\text { Refiere al deseo de estar juntos y } \\
\text { compartir actividades. }\end{array}$ & $\begin{array}{l}\text { Estamos siempre juntos, siempre quiere } \\
\text { jugar con vos y vos siempre con él. }\end{array}$ \\
\hline Conflicto & $\begin{array}{l}\text { Refiere la presencia de conflicto y su } \\
\text { resolución, así como la ausencia del } \\
\text { conflicto. }\end{array}$ & $\begin{array}{l}\text { No importa cuántas veces peleas... un amigo } \\
\text { siempre te perdona. }\end{array}$ \\
\hline Fraternidad & $\begin{array}{l}\text { Refiere sentir a su amigo como un } \\
\text { hermano/a. }\end{array}$ & $\begin{array}{l}\text { A una mejor amiga la querés como } \\
\text { hermana, la podría adoptar. }\end{array}$ \\
\hline Fidelidad & $\begin{array}{l}\text { Refiere a la lealtad que se brinda más } \\
\text { allá de las adversidades. }\end{array}$ & $\begin{array}{l}\text { El mejor amigo está en las buenas y en las } \\
\text { malas. }\end{array}$ \\
\hline Semejanza & $\begin{array}{l}\text { Refiere a las similitudes en el vínculo } \\
\text { amical. }\end{array}$ & $\begin{array}{l}\text { Jugamos a lo mismo, nos gusta la misma } \\
\text { comida. }\end{array}$ \\
\hline Confianza & $\begin{array}{l}\text { El niño/a puede contar lo que le pasa, } \\
\text { sintiéndose cómodo y libre y seguro de } \\
\text { que su amigo sabe guardar un secreto. }\end{array}$ & $\begin{array}{l}\text { Podemos contarle lo que nos pasa y no anda } \\
\text { contando tus cosas. }\end{array}$ \\
\hline Ayuda & $\begin{array}{l}\text { Es el apoyo que se da al mejor amigo, } \\
\text { sea frente a conflictos de tipo material } \\
\text { o emocional. }\end{array}$ & $\begin{array}{l}\text { Te ayuda con los problemas, a no tener } \\
\text { miedo, a no pelear, porque si te insultan él } \\
\text { te dice "no te enojes", "no hagas caso" y te } \\
\text { aconseja asi no das importancia. }\end{array}$ \\
\hline $\begin{array}{l}\text { Emociones } \\
\text { Positivas }\end{array}$ & $\begin{array}{l}\text { Mención a emociones positivas } \\
\text { relacionadas con la amistad }\end{array}$ & $\begin{array}{l}\text { Un amigo te sonrie y vos le sonreís. Se lo } \\
\text { ama. }\end{array}$ \\
\hline $\begin{array}{l}\text { Aspectos } \\
\text { Morales }\end{array}$ & $\begin{array}{l}\text { Mención a cuestiones y juicios } \\
\text { morales en la amistad. }\end{array}$ & $\begin{array}{l}\text { Tiene códigos, respeto, no está con el novio } \\
\text { de la amiga. }\end{array}$ \\
\hline
\end{tabular}

Al comparar las categorías desde $4^{\circ}$ a $7^{\circ}$ grado, se aprecia que algunas categorías son recurrentes en todos los grados, mientras otras no. Así, se identifica que las categorías compañerismo, conflicto, fidelidad, confianza, ayuda y emociones positivas y aspectos morales emergen en el discurso de todos los niños y las niñas desde $4^{\circ}$ a $7^{\circ}$ grado. Sin embargo, la categoría semejanza solo emerge en $4^{\circ}$ grado y la categoría fraternidad en $7^{\circ}$ grado.

Por otra parte, si bien las categorías mencionadas emergen en todos los grados desde $4^{\circ}$ a $7^{\circ}$ grado, se observa que el contenido, sentido y significado en las narrativas difiere según el grado. Por ejemplo, en el caso de la categoría compañerismo, los escolares de $4^{\circ}$ y $5^{\circ}$ grado describen al compañerismo como aquellas actividades basadas en el compartir juguetes, actividades y tareas. Se focalizan en aspectos concretos y tangibles: "...mi mejor amiga me invita a comer, a tomar el té..." (niña, 9 años, $4^{\circ}$ grado); “...estamos siempre juntos, juega con vos, te hace reír, siempre quiere jugar con vos y vos siempre con él..." (varón, 10 años, $5^{\circ}$ grado). Sin embargo, en $6^{\circ}$ y $7^{\circ}$ grado, las expresiones de los niños y las niñas ya no hacen referencia a objetos o situaciones concretas y el compañerismo comienza a centrarse en cuestiones insustanciales o poco tangibles relacionadas con los aspectos vinculares como, por ejemplo: " ...una mejor amiga está con vos siempre, te banca en todas...” (niña, 13 años, $7^{\circ}$ grado); “...lindo, porque es con quien bablas de todo..." (niña, 11 años, $6^{\circ}$ grado). 
Del mismo modo, si bien la categoría fidelidad emerge en todos los grados, se observan diferencias en cuanto al contenido, sentido y significado en los diferentes grados. Nuevamente se observa cómo la fidelidad aparece relacionada con cuestiones de índole material, tangible y concreta en la narrativa de los escolares de $4^{\circ}$ grado: “... una mejor amiga, no te deja plantada por otra que tiene más juguetes que vos..." (niña, 9 años, $4^{\circ}$ grado). Sin embargo, en el discurso de los escolares de $5^{\circ}$ a $7^{\circ}$ grado, la fidelidad se relaciona con la compañía y la cercanía en situaciones de adversidad. Por ejemplo: “...un mejor amigo está siempre en las buenas y en las malas...” (varón, 10 años, $5^{\circ}$ grado); “...un mejor amigo está en un momento difícil, cuando se muere mi abuela" (niña, 11 años, $6^{\circ}$ grado).

La categoría ayuda se refiere al apoyo que se da al mejor amigo sea frente a conflictos de tipo material o emocional. En la narrativa de los escolares se destaca el afrontamiento y soporte afectivo en las relaciones interpersonales. Así, un varón de 11 años, de $6^{\circ}$ grado refiere: "... un mejor amigo te ayuda con los problemas, a no tener miedo, a no pelear, porque si te insultan él te dice no te enojes, no hagas caso y te aconseja...".

Otra de las categorías que emergió en todos los grados fue la categoría conflicto, la cual se subdividió en dos subcategorías: ausencia de conflicto y reconciliación. El contenido, sentido y significado de las narrativas es el mismo en todos los grados. Los escolares refieren la ausencia del conflicto o la búsqueda de reconciliación, si lo hubiera. Por ejemplo, un varón de 9 años y de $4^{\circ}$ grado comenta: “... no importa cuántas veces te peleas un amigo siempre te perdona..."; “... a veces discutimos con mi amigo, nos peleamos, pero hablamos y está todo bien...” (varón, 12 años, $7^{\circ}$ grado).

La categoría semejanzas solo emergió en el discurso de los niños de $4^{\circ}$, no así en $5^{\circ}, 6^{\circ}$ y $7^{\circ}$ grado. Para ilustrar, está el caso de una niña de 9 años en $4^{\circ}$ grado dice: “... jugamos a lo mismo, nos gusta la misma comida, hacemos lo mismo...". Del mismo modo, la categoría fraternidad solo emergió en $7^{\circ}$ grado. Tal es el caso de una niña de 12 años en $7^{\circ}$ grado dice: “... una mejor amiga es a quien la querés como una hermana, la podría adoptar...”.

En cuanto a la categoría referida a aspectos emocionales, los niños y niñas de todos los grados mencionaron emociones de tono positivo. Por ejemplo: “... un mejor amigo es amable, cariñoso..." (varón, 10 años, $5^{\circ}$ grado); “... mi mejor amiga es buena, sabe lo que siento, lo que me pasa, no es egoísta..." (niña, 11 años, $6^{\circ}$ grado).

Igual que en el caso anterior, la categoría aspecto moral se presentó desde $4^{\circ}$ a $7^{\circ}$ grado; sin embargo, el contenido, la manifestación y la expresión del comportamiento moral en las relaciones de amistad se va complejizando en $6^{\circ}$ y $7^{\circ}$ grado. Al respecto, un varón de 10 años en $5^{\circ}$ dice: “...un mejor amigo te deja espacio, te respeta...”; “... un mejor amigo no tendría que ser buchón...” (varón, 11 años, $6^{\circ}$ grado). Al pasar a séptimo grado, el tema de la traición aparece en el marco de las relaciones románticas (aspecto que no surge en los grados anteriores); por ejemplo, una niña de 13 años de edad, de $7^{\circ}$ grado refiere: “...una mejor amiga tiene códigos, respeto, no está con el novio de la mejor amiga...".

Del mismo modo, que el objetivo número 1 consistió en caracterizar al mejor amigo, el objetivo número 2 exploró la comprensión que tienen los escolares de $4^{\circ}, 5^{\circ}, 6^{\circ}$ y $7^{\circ}$ grado sobre el concepto del mejor amigo como factor protector frente a situaciones 
de maltrato entre pares. En esta oportunidad, del discurso emergieron cinco categorías, tres de las cuales se focalizaron en el rol protector que desempeña el mejor amigo y cuya estrategia va dirigida al agresor; otra categoría tiene que ver con el rol protector pero, en esta oportunidad, la estrategia va dirigida a la víctima; finalmente, una última categoría cuya función de protección se manifiesta en recurrir a una autoridad (docente). Los resultados se resumen en la tabla 2.

En cuanto a las estrategias dirigidas al agresor, se observa en la tabla 2 la categoría de "rol protector: manifestaciones de violencia hacia el agresor". Esta emergió en todos los escolares de $4^{\circ}$ a $7^{\circ}$ grado y fue definida como aquellas manifestaciones de violencia hacia el agresor en caso de maltrato e intimidación hacia el mejor amigo. Algunas de las verbalizaciones fueron: "... Cuando lo tratan mal a mi amigo lo defiendo a veces con piñas..." (niño, 9 años, $4^{\circ}$ grado); “...si le hacen algo a mi amiga los insulto para defenderla...” (niña, 11 años, $6^{\circ}$ grado).

Otra de las categorías que surgió hacia el agresor fue: "búsqueda de diálogo con el agresor". Esta tuvo su foco en intentar hacer reflexionar al agresor acerca de su comportamiento agresivo. Por ejemplo, un varón de 9 años de $4^{\circ}$ grado refiere: “. .. si lo tratan mal a mi amigo yo le digo ¿̇por qué haces eso?, ¿̇or qué lo tratas así?, no ves que se siente mal...”.

Tabla 2

Esquema de categorías empleadas en el análisis del mejor amigo como factor protector frente a situaciones de maltrato entre pares

\begin{tabular}{|c|c|c|}
\hline Categoría & Definición & Ejemplo \\
\hline $\begin{array}{l}\text { Rol Protector: } \\
\text { Manifestaciones de } \\
\text { violencia hacia el } \\
\text { agresor }\end{array}$ & $\begin{array}{l}\text { Se refiere a todos aquellos comportamientos } \\
\text { agresivos, físicos y/o verbales, orientados a } \\
\text { proteger al mejor amigo de situaciones de } \\
\text { intimidación. }\end{array}$ & $\begin{array}{l}\text { Cuando lo tratan mal a mi } \\
\text { defiendo a veces con piñas. }\end{array}$ \\
\hline $\begin{array}{l}\text { Rol Protector: } \\
\text { Búsqueda de diálogo } \\
\text { con el agresor. }\end{array}$ & $\begin{array}{l}\text { Se consideran todas aquellas } \\
\text { verbalizaciones en las cuales el mejor } \\
\text { amigo intenta dialogar con el agresor a } \\
\text { fin de evitar o amortiguar los efectos de } \\
\text { la intimidación hacia su mejor amigo. }\end{array}$ & $\begin{array}{l}\text { Si lo tratan mal a mi amigo yo le } \\
\text { dijo por qué haces eso?, por qué lo } \\
\text { tratas asi?, no ves que se siente mal. }\end{array}$ \\
\hline $\begin{array}{l}\text { Rol Protector: } \\
\text { Contención afectiva } \\
\text { por medio de la } \\
\text { palabra hacia la } \\
\text { víctima. }\end{array}$ & $\begin{array}{l}\text { Son aquellas verbalizaciones en las } \\
\text { cuales se denota contención afectiva, } \\
\text { soporte hacia la víctima por parte del } \\
\text { mejor amigo. }\end{array}$ & $\begin{array}{l}\text { Mi amigo me da palabras de aliento } \\
\text { diciendo que me tranquilice, que no } \\
\text { le haga caso, que es un tonto, que yo } \\
\text { valgo más. }\end{array}$ \\
\hline $\begin{array}{l}\text { Rol Protector: } \\
\text { Estrategia } \quad \text { de } \\
\text { ignorar al agresor o } \\
\text { agresores. }\end{array}$ & $\begin{array}{l}\text { Son todos aquellos comportamientos } \\
\text { en los cuales quienes han sido víctimas } \\
\text { de intimidación implementan como } \\
\text { estrategias de afrontamiento la retirada } \\
\text { e indiferencia hacia el agresor, a fin de }\end{array}$ & $\begin{array}{l}\text { Nosotros nos van } \\
\text { cerca de quien no. }\end{array}$ \\
\hline $\begin{array}{l}\text { Rol Protector: } \\
\text { Búsqueda de apoyo } \\
\text { en el docente. }\end{array}$ & $\begin{array}{l}\text { Son los comportamientos en los cuales } \\
\text { se recurre a la autoridad. }\end{array}$ & $\begin{array}{l}\text { seño para que } \\
\text { eleas. }\end{array}$ \\
\hline
\end{tabular}


La tercera de las categorías que emergió hacia el agresor fue "el rol protector: estrategia de ignorar al agresor". Así, una niña de 10 años de $4^{\circ}$ grado refiere: “. .. nosotras con mi amiga nos vamos, para no estar cerca de quien nos molesta, así no le damos importancia...". Sin embargo, en los grados superiores como $7^{\circ}$ grado además de implementar la retirada (como estrategia frente al maltrato), el mejor amigo brinda explicaciones y atribuciones de por qué es preferible irse o ignorarlo. De este modo, un varón de 12 años de $7^{\circ}$ grado menciona “... Yo le digo a mi amigo que nos vayamos, que no le demos importancia, que ellos lo que quieren es vernos mal...".

La categoría que emergió del discurso como "rol protector: contención afectiva por medio de la palabra hacia la víctima" predominó en $4^{\circ}$ y $5^{\circ}$ grado, mas no así en $6^{\circ}$ y $7^{\circ}$ grado. Un ejemplo de dicha categoría es el siguiente: “... mi amiga me da palabras de aliento diciendo que me tranquilice, que no le haga caso, que es un tonto, que yo valgo más” (niña, 9 años, $4^{\circ}$ grado). Un escolar de $5^{\circ}$ grado refiere: "mi amigo me dice cosas lindas para que no me ponga triste" (niña, 10 años, $5^{\circ}$ grado).

Otra de las categorías que emergió del discurso de los niños y las niñas frente a situaciones de maltrato por parte de los pares fue la recurrencia a la autoridad. Dicha categoría solo fue descrita por los niños y las niñas de $4^{\circ}$ grado. Al respecto, uno de los escolares de $4^{\circ}$ grado refirió: "mi amigo busca a la seño para que diga "basta", basta de peleas" (varón, 9 años).

\section{Discusión}

Los niños y las niñas definieron la amistad con el mejor amigo a partir de nueve categorías que emergieron de su propio discurso: compañerismo, fraternidad, confianza, ayuda, semejanzas, presencia/resolución de conflictos y características emocionales y morales. Tales características y definiciones van en línea con las estudiadas hasta ahora en torno a la amistad en la infancia (Kancyper, 2014; Papalia, Wendkos, \& Duskin, 2010; Rubin \& Bowker, en prensa).

No obstante, se observaron diferencias a la hora de conceptualizar una misma característica de la amistad. Tales diferencias podrían deberse a cuestiones propias del desarrollo cognitivo y socioemocional en la infancia. El concepto de amistad que poseen los niños y las niñas y la manera en cómo se relacionan con sus mejores amigos cambian con la edad, lo cual es reflejo de su crecimiento (Papalia, Wendkos, \& Duskin, 2010).

Tal diferencia también podría deberse a lo planteado por Hartup (2009), quien refiere que la reciprocidad característica básica de los vínculos de amistad, en todas las etapas del desarrollo, se manifiesta en diferentes comportamientos sociales según la edad. Desde la Teoría de Selman (1980), tales diferencias y características de la amistad se comprenden en el marco de la etapa dos y tres descritas por Selman y que permiten entender la conceptualización de la amistad con base en la reciprocidad y a la toma de perspectiva.

Por otra parte, los niños y las niñas conceptualizaron propósitos morales al definir la amistad, tales respuestas van en línea con lo planteado por Bukowski y Sippola (1996), quienes refieren que la amistad ha sido frecuentemente caracterizada como una relación moral especial que fomenta la bondad moral en las personas y, en este sentido, la amistad y la moral están inextricablemente interrelacionadas. 
Otra de las categorías que emergió en este trabajo al caracterizar la amistad con un mejor amigo fueron las emociones positivas. Los niños y las niñas describieron el cariño, la ternura, la diversión, la amabilidad, la alegría, la solidaridad y la felicidad como emociones que son parte del vínculo amical. Tales resultados van en línea con lo planteado por Demir et al. (2013); Holder y Coleman, (2008); Saldarriaga, Bukowski y Greco, (2015), quienes plantean que hay una relación bidireccional entre felicidad y amistad.

Con respecto al objetivo 2, las categorías que emergen tienen que ver con estrategias de soporte y ayuda dirigidas al agresor, a la víctima y de recurrencia a la autoridad. Tales resultados van en línea con investigaciones previas que refieren que los agresores podrían temer represalias o comportamientos de exclusión por parte de los amigos de las víctimas y, en ese sentido funcionar, como un factor protector. Además, los niños y las niñas que tienen amigos suelen estar en compañía de otros, por lo que no son elegidos como víctimas (Saldarriaga, Bukowski, \& Greco, 2015).

Los resultados van de acuerdo con el modelo de Sullivan (1953) acerca de la amistad como factor protector y soporte en situaciones de riesgo. Asimismo, los hallazgos concuerdan con investigaciones previas acerca de los vínculos de amistad como un factor protector frente a situaciones de intimidación y maltrato entre pares (Bukowski, Motzoi, \& Meyer, 2009; Hodges, Boiven, Vitaro, \& Bukowski, 1999).

Finalmente, los niños y las niñas que tienen amigos reciben consejos de ellos sobre cómo resolver conflictos o enfrentar las amenazas. De hecho, en este trabajo la estrategia de soporte dirigida a la víctima tiene que ver con ese soporte afectivo y de validación del sí mismo frente al maltrato por parte de sus pares, aspectos que van en línea con la teoría de Sullivan (1953).

\section{Conclusiones}

Este trabajo permite pensar a los vínculos de amistad en la infancia como un recurso de índole psicosocial que cumple la función de factor protector frente a situaciones de adversidad. Asimismo, posibilita un desarrollo positivo en la infancia, relacionado con la experiencia de emociones positivas, el bienestar y la validación del sí mismo.

Además, la naturaleza y dinámica de los vínculos de amistad en la infancia son un escenario propicio para el despliegue de habilidades cognitivas y socioafectivas; para el desarrollo de valores y juicios morales; para el entrenamiento y desarrollo de habilidades de solución de conflictos interpersonales y para el desarrollo de sentimientos como el amor, la lealtad, la fidelidad, el compañerismo, la gratitud, la empatía y el sentido del humor.

Si bien en este trabajo el total de los niños y niñas participantes refirieron tener un mejor amigo/a, es impostergable el estudio y preocupación por aquellos niños y niñas que tienen dificultades para interactuar con los otros y construir y consolidar vínculos de amistad. Si se piensa que relacionarse con los pares conlleva a un desarrollo holístico que propicia el desarrollo de recursos y ajuste psicosocial, es necesario entonces, abordar aquellos niños y niños con dificultades en las relaciones interpersonales e implementar intervenciones que promuevan dichos vínculos. 
El uso de la metodología cualitativa para la indagación y exploración de los vínculos de amistad en la infancia permite diseñar pautas de intervención desde la voz de los propios niños y niñas y adecuarlos a los contextos de referencias.

Finalmente, se mencionan algunas implicaciones prácticas para el desarrollo de los vínculos de amistad en la infancia en el contexto escolar. Como sugerencia, el juego es uno de los elementos a fomentar cuando se trata de promocionar las interacciones sociales entre pares y los vínculos de amistad. Se recomiendan juegos en compañía y de tipo cooperativo que permitan la puesta en acción del compañerismo, la solidaridad y la reciprocidad, evitando el juego paralelo y solitario.

En cuanto a la intervención propiamente dicha, García Bacete, Rubio Barreda, Milian Rojas y Marande Perrin (2013) destacan diferentes modalidades de intervención en la escolaridad primaria. Una de ellas es junto a los padres de los niños y las niñas que presenten dificultades en la interacción social.

El objetivo de tales intervenciones es que los padres puedan facilitar el valor y aprendizaje de la amistad. Otro tipo de intervenciones está focalizado en el grupo de amigos. En este caso, el grupo de amistad suele estar formado por un estudiante con dificultades sociales y un adulto facilitador. Su función principal es proporcionar oportunidades para practicar habilidades de competencia social.

Por otra parte, el programa de terapia entre iguales (Selman, 1980) consiste en sesiones de juego supervisadas por un adulto en la cual los dos escolares tienen dificultades de relación. Dicha intervención ha sido evaluada favorablemente; sin embargo, se recalca que todo tipo de intervención debe ser considerada teniendo en cuenta la subjetividad de cada caso en particular y las características contextuales.

\section{Referencias}

Ainsworth, M. S. (1989). Attachments beyond infancy. American Psychologist, 44(4), 709-716. doi: 10.1037/0003-066X.44.4.709

Blos, P. (1979). The adolescent passage. New York: International Univerities Press.

Bowlby, J. (1958). The Nature of child's tie to his mother. International Journal of PsychoAnalysis, (30), 350-373.

Bukowski, W. M., Hoza, B., \& Boivin, M. (1994). Measuring friendship quality during pre and early adolescence: The Development and psychometric properties of the friendship qualities scale. Journal of Social and Personal Relationships, 11(3), 471-484. doi: $10.1177 / 0265407594113011$

Bukowski, W. M., Motzoi, C., \& Meyer, F. (2009). Friendship as process, function, and outcome. In K. H. Rubin, W. Bukowski, \& B. Laursen (Eds.), Handbook of peer interactions, relationships, and groups (pp. 217-231). Nueva York: The Guilford Press.

Bukowski, W. M. \& Sippola, L. K. (2005). Friendship and development: Putting the most human relationship in its place. New directions for children and adolescent development. Wiley Periodical, (109), 91-98. doi: 10.1002/cd.141 
Cohen, J. (1960). A coefficient of agreement for nominal scales. Educational and Psychological Measurement, 20(1), 37-46. doi: 10.1177/001316446002000104

Collins, W. A., Welsh, D. P., \& Furman, W. (2009). AdolescentRomantic Relationships. Annual Review of Psychology, 60, 631-652. doi: 10.1146/annurev.psych.60.110707.163459

Demir, M., Orthel, H., \& Andelin, A. K. (2013). Friendship and happiness. In S. David, I. Boniwell, \& A. C. Ayers (Eds.), The Oxford handbook of happiness (pp. 860-870). Oxford: Oxford University Press.

Demir, M. \& Özdemir, M. (2010). Friendship, need satisfaction and happiness. Journal of Happiness Study, 11(2), 243-259. doi: 10.1007/s10902-009-9138-5

Fuentes Rebollo, M. J. \& Melero Zabal, M. A. (1992). Las amistades infantiles: desarrollo, funciones y pautas de intervención en la escuela. Revista Investigación en la Escuela, (16), 55-67.

Freud, S. (1997). Ensayos XXVI al XLV. En L. Lopez Ballesteros y de Torres (Traduc.), Obras Completas: Sigmund Freud, (Tomo 4: 1905-1910). Madrid: Biblioteca Nueva.

García Bacete, F. J., Rubio Barrerda, A., Milan Rojas, I., \& Marande Perrín G. (2013). El aprendizaje de la amistad en la educación primaria. Un procedimiento intensivo para ayudar a los niños rechazados a hacer amigos. Apuntes de Psicología, 31, 155-163.

Glaser B. \& Strauss, A. (1967). The discovery of grounded theory: Strategies for qualitative research. New York: Aldine Publishing Company.

Greco, C. \& Ison, M. S. (2014). What makes you happy?: Appreciating the reasons that bring happiness to Argentine children living in vulnerable social contexts. Journal of Latino-Latin American Studies 6(1), 4-18. doi: 10.18085/1las.6.1.q8158245p8267638

Hartup, W. W. (2009). Critical issues and theoretical viewpoints. In K. H. Rubin, W. M. Bukowski, \& B. Laursen (Eds.), Social, emotional, and personality development in context. Handbook of peer interactions, relationships, and groups (pp. 3-19). New York, NY, US: Guilford Press.

Hartup, W. W. \& Stevens, N. (1997). Friendships and adaptation in the life course. Psychological Bulletin, 121(3), 355-370. doi: 10.1037/0033-2909.121.3.355

Hendrick, C. \& Hendrick, S. S., (2000). Close relationships: A sourcebook. Londres: Sage Publications. doi: 10.4135/9781452220437

Hinde, R. (1987). Individuals, relationships, and culture. Links between ethology and the social sciences. Cambridge, USA: Cambridge University Press.

Hodges, E. V. E., Boivin, M., Vitaro, F., \& Bukowski, W. M. (1999). The power of friendship: Protection against an escalating cycle of peer victimization. Developmental Psychology, 35(1), 94-101. doi: 10.1037/0012-1649.35.1.94

Holder, M. D., \& Coleman, B. (2008). The contribution of temperament, popularity, and physical appearance to children's happiness. Journal of Happiness Studies, 9(2), 279302. doi: 10.1007/s10902-007-9052-7 
Kancyper, L. (2014). Amistad: una hermandad elegida. Estudio Psicoanalítico. Buenos Aires: Lumen.

Kochenderfer, B. J. \& Ladd, G. (1996). Peer victimization. Cause or consequences of school maladjustment? Child Development, 67(4), 135-1317. doi: 10.1111/j.14678624.1996.tb01797.x

Kornblit, A. L. (2004). Metodología cualitativa en ciencias sociales. Colombia: Biblos.

Landis, J. R. \& Koch, G. G. (1977). The Measurement of observer agreement for categorical data. Biometrics, 33(1), 159-174. doi: 10.2307/2529310

Papalia, D. E, Wendkos, O. S. \& Duskin, F. R. (2010). Human Development. Miami: Dade College.

Potocnjak, M., Berger, C., \& Tomicic, T. (2011). Una aproximación relacional a la violencia escolar entre pares en adolescentes chilenos: perspectiva adolescente. Revista Psykhe, 20(2), 39-52. doi: 10.4067/S0718-22282011000200004

Rubin, K. H. \& Bowker, J. (In press). Friendship. In M. Bornstein, Arterberry, M.E., Fingerman, K.L., \& Lansford, J. E. (Eds.). The Sage encyclopedia of lifespan buman development. Thousand Oaks, CA: Sage.

Rubin, K. H., Bukowski, W. M., \& Parker, J. G. (2007). Peer interactions, relationships, and groups. Handbook of Child Psychology. doi: 10.1002/9780470147658.chpsy0310

Rubin, K. H., Coplan, R., Chen, X., Bowker, J., \& McDonald, K. L. (2011). Peer relationships in childhood. In M. H. Bornstein \& M. E. Lamb (Eds.), Developmental science: An advanced textbook (pp. 519-570). New York: Psychology.

Saldarriaga L., Bukowski W., \& Greco C. (2015). Friendship and Happiness: A Bidirectional Dynamic Process. In: Demir M. (Eds.) Friendship and Happiness. Dordrecht: Springer. doi: 10.1007/978-94-017-9603-3_4

Selman, R. L. (1980). The growth of interpersonal understanding. Nueva York: Academic Press.

Sullivan, H. S. (1953). The interpersonal theory of psychiatry. Nueva York: Norton.

Thibaut, J. W. \& Kelley, H. H. (1959). The social psychology of groups. New York: John Wiley \& Sons.

Vitaro, F., Boivin, M. \& Bukowski, M. W. (2009). The role of friendship in child and adolescent psychosocial development. In: K. Rubin, W. Bukowski, \& B. Laursen (Eds.), Handbook of peer interactions, relationships and groups (pp. 568-588). New York: The Guildford Press.

Villalobos, C. (2015). Estudio de las relaciones de amistad adolescente en contextos educativos de diversidad cultural (tesis de posgrado) Universitat de Barcelona, Barcelona España. Recuperado de http://hdl.handle.net/2445/116086 